\title{
On a Multipoint Boundary Value Problem for a Fractional Order Differential Inclusion on an Infinite Interval
}

\author{
Nemat Nyamoradi, ${ }^{1}$ Dumitru Baleanu, ${ }^{2,3,4}$ and Ravi P. Agarwal ${ }^{5,6}$ \\ ${ }^{1}$ Department of Mathematics, Faculty of Sciences, Razi University, Kermanshah 67149, Iran \\ ${ }^{2}$ Department of Mathematics and Computer Sciences, Faculty of Art and Sciences, Cankaya University, 06530 Ankara, Turkey \\ ${ }^{3}$ Institute of Space Sciences, P.O. BOX MG-23, 76900 Magurele, Bucharest, Romania \\ ${ }^{4}$ Department of Chemical and Materials Engineering, Faculty of Engineering, King Abdulaziz University, P.O. Box 80204, \\ Jeddah 21589, Saudi Arabia \\ ${ }^{5}$ Department of Mathematics, Texas A \& M University-Kingsville, 700 University Boulevard, Kingsville, USA \\ ${ }^{6}$ Department of Mathematics, King Abdulaziz University, P.O. Box 80204, Jeddah 21589, Saudi Arabia
}

Correspondence should be addressed to Dumitru Baleanu; dumitru@cankaya.edu.tr

Received 12 January 2013; Accepted 22 January 2013

Academic Editor: José Tenreiro Machado

Copyright (c) 2013 Nemat Nyamoradi et al. This is an open access article distributed under the Creative Commons Attribution License, which permits unrestricted use, distribution, and reproduction in any medium, provided the original work is properly cited.

We investigate the existence of solutions for the following multipoint boundary value problem of a fractional order differential inclusion $D_{0^{+}}^{\alpha} u(t)+F\left(t, u(t), u^{\prime}(t)\right) \ni 0,0<t<+\infty, u(0)=u^{\prime}(0)=0, D^{\alpha-1} u(+\infty)-\sum_{i=1}^{m-2} \beta_{i} u\left(\xi_{i}\right)=0$, where $D_{0^{+}}^{\alpha}$ is the standard Riemann-Liouville fractional derivative, $2<\alpha<3,0<\xi_{1}<\xi_{2}<\cdots<\xi_{m-2}<+\infty$, satisfies $0<\sum_{i=1}^{m-2} \beta_{i} \xi_{i}^{\alpha-1}<\Gamma(\alpha)$, and $F$ : $[0,+\infty) \times \mathbb{R} \times \mathbb{R} \rightarrow \mathscr{P}(\mathbb{R})$ is a set-valued map. Several results are obtained by using suitable fixed point theorems when the right hand side has convex or nonconvex values.

\section{Introduction}

In this paper, we will consider the existence of solutions for the following multipoint boundary value problem of a fractional order differential inclusion

$$
\begin{gathered}
D_{0^{+}}^{\alpha} u(t)+F\left(t, u(t), u^{\prime}(t)\right) \ni 0, \quad 0<t<+\infty, \\
u(0)=u^{\prime}(0)=0, \quad D^{\alpha-1} u(+\infty)-\sum_{i=1}^{m-2} \beta_{i} u\left(\xi_{i}\right)=0,
\end{gathered}
$$

where $D_{0^{+}}^{\alpha}$ is the standard Riemann-Liouville fractional derivative, $2<\alpha<3,0<\xi_{1}<\xi_{2}<\cdots<\xi_{m-2}<+\infty$, satisfies $0<\sum_{i=1}^{m-2} \beta_{i} \xi_{i}^{\alpha-1}<\Gamma(\alpha)$, and $F:[0,+\infty) \times \mathbb{R} \times \mathbb{R} \rightarrow$ $\mathscr{P}(\mathbb{R})$ is a set-valued map.

The present paper is motivated by a recent paper of Liang and Zhang [1], where it is considered problem (1) with $F$ single valued, and several existence results are provided.

Fractional differential equations have been of great interest recently. This is because of both the intensive development of the theory of fractional calculus itself and the applications of such constructions in various scientific fields such as physics, mechanics, chemistry, and engineering. For details, see $[2-4]$ and the references therein.

The existence of solutions of initial value problems for fractional order differential equations has been studied in the literature [5-17] and the references therein. The study of fractional differential inclusions was initiated by El-Sayed and Ibrahim [18]. Also, recently, several qualitative results for fractional differential inclusions were obtained in [19-23] and the references therein.

The aim here is to establish existence results for problem (1) when the right hand side is convex as well as nonconvex valued. In the first result (Theorem 21), we consider the case when the right hand side has convex values and prove an existence result via nonlinear alternative for Kakutani maps. In the second result (Theorem 25), we will use the fixed point theorem for contraction multivalued maps according to Covitz and Nadler. The paper is organized as follows. 
In Section 2 we recall some preliminary facts that we need in the sequel, and in Section 3 we prove our main results. Finally, in Section 4, an example is given to demonstrate the application of one of our main results.

\section{Preliminaries}

In this section, we present some notations and preliminary lemmas that will be used in the proof of the main result.

Let $(X, d)$ be a metric space with the corresponding norm $\|\cdot\|$ and let $I=[0,+\infty)$. We denote by $\mathscr{L}(I)$ the $\sigma$-algebra of all Lebesgue measurable subsets of $I$, by $\mathscr{B}(X)$ the family of all nonempty subsets of $X$, and by $\mathscr{P}(X)$ the family of all Borel subsets of $X$. If $A \subset I$ then $\chi_{A}: I \rightarrow\{0,1\}$ denotes the characteristic function of $A$. For any subset $A \subset X$, we denote by $\bar{A}$ the closure of $A$.

Recall that the Pompeiu-Hausdorff distance of the closed subsets $A, B \subset X$ is defined by the following:

$$
\begin{gathered}
d_{H}(A, B)=\max \left\{d^{*}(A, B), d^{*}(B, A)\right\}, \\
d^{*}(A, B)=\sup \{d(a, B), a \in A\},
\end{gathered}
$$

where $d(x, B)=\inf _{y \in B} d(x, y)$. Define

$$
\begin{gathered}
\mathscr{P}(X)=\{Y \subset X: Y \neq \emptyset\}, \\
\mathscr{P}_{b}(X)=\{Y \in \mathscr{P}(X): Y \text { bounded }\}, \\
\mathscr{P}_{c l}(X)=\{Y \in \mathscr{P}(X): Y \text { closed }\} \\
\mathscr{P}_{c p}(X)=\{Y \in \mathscr{P}(X): Y \text { compact }\}, \\
\mathscr{P}_{c v}(X)=\{Y \in \mathscr{P}(X): Y \text { convex }\} .
\end{gathered}
$$

Also, we denote by $C(I, X)$ the Banach space of all continuous functions $x:[0,+\infty) \rightarrow X$ endowed with the norm $|x|_{c}=\sup _{t \in[0,+\infty)}|x(t)|$ and by $L^{1}([0,+\infty), X)$ the Banach space of all (Bochner) integrable functions $x:[0,+\infty) \rightarrow X$ endowed with the norm $|x|_{1}=\int_{[0,+\infty)}|x(t)| d t$.

Let $\left(X, d_{1}\right)$ and $\left(Y, d_{2}\right)$ be two metric spaces. If $T: X \rightarrow$ $\mathscr{P}(X)$ is a set-valued map, then a point $x \in X$ is called a fixed point for $T$ if $x \in T(x)$. T is said to be bounded on bounded sets if $T(B):=\cup_{x \in B} T(x)$ is a bounded subset of $X$ for all bounded sets $B$ in $X$. $T$ is said to be compact if $T(B)$ is relatively compact for any bounded sets $B$ in $X$. T is said to be totally compact if $\overline{T(X)}$ is a compact subset of $X . T$ is said to be upper semicontinuous if for any open set $D \subset X$, the set $\{x \in X: T(x) \subset D\}$ is open in $X$. T is called completely continuous if it is upper semicontinuous and, for every bounded subset $A \subset X, T(A)$ is relatively compact. It is well known that a compact set-valued map $T$ with nonempty compact values is upper semicontinuous if and only if $T$ has a closed graph.

We define the graph of $T$ to be the set $\operatorname{Gr}(T)=\{(x, y) \in$ $X \times Y, y=T(x)\}$ and recall a useful result regarding connection between closed graphs and upper semicontinuity.

Lemma 1 (see [24, Proposition 1.2]). If $T: X \rightarrow \mathscr{P}_{c l}(Y)$ is upper semicontinuous, then $G r(T)$ is a closed subset of $X \times Y$, that is, for every sequence $\left\{x_{n}\right\}_{n \in \mathbb{N}} \subset X$ and $\left\{y_{n}\right\}_{n \in \mathbb{N}} \subset Y$, if when $n \rightarrow \infty, x_{n} \rightarrow x_{*}, y_{n} \rightarrow y_{*}$, and $y_{n} \in T\left(x_{n}\right)$, then $y_{*} \in T\left(x_{*}\right)$. Conversely, if $T$ is completely continuous and has a closed graph, then it is upper semicontinuous.

For the convenience of the reader, we present here the following nonlinear alternative of the Leray-Schauder type and its consequences.

Theorem 2 (nonlinear alternative for Kakutani maps [25]). Let $X$ be a Banach space, $C$ a closed convex subset of $X$, $U$ an open subset of $C$, and $0 \in U$. Suppose that $T$ : $\bar{U} \rightarrow \mathscr{P}_{c l, c v}(C)$ is an upper semicontinuous compact map; here $\mathscr{P}_{c l, c v}(C)$ denotes the family of nonempty, compact convex subsets of $C$. Then, either

(i) Thas a fixed point in $U$ or

(ii) there is a $u \in \partial U$ and $\lambda \in(0,1)$ with $u \in \lambda T(u)$.

Definition 3. The multifunction $T: X \rightarrow \mathscr{P}(X)$ is said to be lower semicontinuous if for any closed subset $C \subset X$, the subset $\{s \in X: T(s) \subset C\}$ is closed.

If $F:[0,+\infty) \times \mathbb{R} \times \mathbb{R} \rightarrow \mathscr{P}(\mathbb{R})$ is a set-valued map with compact values and $x \in C([0,+\infty), \mathbb{R})$, we define

$$
\begin{aligned}
S_{F}(x):=\{f & \in L^{1}([0,+\infty), \mathbb{R}): f(t) \\
& \left.\in F\left(t, x(t), x^{\prime}(t)\right) \text { a.e. }[0,+\infty)\right\} .
\end{aligned}
$$

Then, $F$ is of a lower semicontinuous type if $S_{F}(\cdot)$ is a lower semicontinuous with closed and decomposable values.

Theorem 4 (see [26]). Let $S$ be a separable metric space and $G: S \rightarrow \mathscr{P}\left(L^{1}([0,+\infty), \mathbb{R})\right)$ be a lower semicontinuous setvalued map with closed decomposable values. Then $G$ has a continuous selection (i.e., there exists a continuous mapping $g: S \rightarrow L^{1}([0,+\infty), \mathbb{R})$ such that $g(s) \in G(s)$ for all $\left.s \in S\right)$.

Definition 5. Consider the following.

(i) A set-valued map $G:[0,+\infty) \rightarrow \mathscr{P}(\mathbb{R})$ with nonempty compact convex values is said to be measurable if for any $x \in \mathbb{R}$ the function $t \rightarrow d(x, G(t))$ is measurable.

(ii) A set-valued map $F:[0,+\infty) \times \mathbb{R} \times \mathbb{R} \rightarrow \mathscr{P}(\mathbb{R})$ is said to be Carathéodory if $t \rightarrow F(t, x, y)$ is measurable for all $x, y \in \mathbb{R}$ and $(x, y) \rightarrow F(t, x, y)$ is upper semicontinuous for almost all $t \in[0,+\infty)$.

(iii) $F$ is said to be $L^{1}$-Carathéodory if for any $l>0$ there exists $h_{l} \in L^{1}([0,+\infty), \mathbb{R})$ such that $\sup \{|v|: \quad v \in$ $F(t, x, y)\} \leq h_{l}(t)$ a.e. $[0,+\infty) ; \forall x, y \in \mathbb{R}$.

Finally, the following results are easily deduced from the theoretical limit set properties. 
Lemma 6 (see [27, Lemma 1.1.9]). Let $\left\{K_{n}\right\}_{n \in \mathbb{N}} \subset K \subset X$ be a sequence of subsets where $K$ is a compact subset of a separable Banach space $X$. Then,

$$
\overline{\mathrm{co}}\left(\limsup _{n \rightarrow \infty} K_{n}\right)=\bigcap_{N>0} \overline{\mathrm{co}}\left(\bigcup_{n \geq N} K_{n}\right),
$$

where $\overline{\mathrm{co}}(A)$ refers to the closure of the convex hull of $A$.

Lemma 7 (see [27, Lemma 1.4.13]). Let $X$ and $Y$ be two metric spaces. If $: X \rightarrow \mathscr{P}_{c p}(Y)$ is an upper semicontinuous, then, for each $x_{0} \in X$,

$$
\limsup _{x \rightarrow x_{0}} G(x)=G\left(x_{0}\right) \text {. }
$$

Definition 8. Let $X$ be a Banach space. A sequence $\left\{x_{n}\right\}_{n \in \mathbb{N}} \subset$ $L^{1}([a, b], X)$ is said to be semicompact if

(a) it is integrably bounded; that is, there exists $q \in$ $L^{1}\left([a, b], \mathbb{R}^{+}\right)$such that

$\left|x_{n}(t)\right|_{E} \leq q(t), \quad$ for a.e. $t \in[a, b]$ and every $n \in \mathbb{N}$,

(b) the image sequence $\left\{x_{n}(t)\right\}_{n \in \mathbb{N}}$ is relatively compact in $E$ for a.e. $t \in[a, b]$.

The following important result follows from the DunfordPettis theorem (see [28, Proposition 4.2.1]).

Lemma 9. Every semicompact sequence $L^{1}([a, b], X)$ is weakly compact in $L^{1}([a, b], X)$.

When the nonlinearity takes convex values, Mazur's Lemma, 1933, may be useful.

Lemma 10 (see [29, Theorem 21.4]). Let E be a normed space and $\left\{x_{k}\right\}_{k \in \mathbb{N}} \subset E$ a sequence weakly converging to a limit $x \in$ $E$. Then, there exists a sequence of convex combinations $y_{m}=$ $\sum_{k=1}^{m} \alpha_{m k} x_{k}$ with $\alpha_{m k}>0$ for $k=1,2, \ldots, m$ and $\sum_{k=1}^{m} \alpha_{m k}=1$ which converges strongly to $x$.

Lemma 11 (see [30]). Let $X$ be defined as before and $M \subset X$. Then $M$ is relatively compact in $X$ if the following conditions hold:

(a) $M$ is uniformly bounded in $X$;

(b) the functions from $M$ are equicontinuous on any compact interval of $[0,+\infty)$;

(c) the functions from $M$ are equiconvergent; that is, for any given $\epsilon>0$, there exists a $T=T(\epsilon)>0$ such that $|f(t)-f(+\infty)|<\epsilon$, for any $t>T, f \in M$.

Definition 12 (see [6]). The Riemann-Liouville fractional integral operator of order $\alpha>0$, of function $f \in L^{1}\left(\mathbb{R}^{+}\right)$, is defined as

$$
I_{0^{+}}^{\alpha} f(t)=\frac{1}{\Gamma(\alpha)} \int_{0}^{t}(t-s)^{\alpha-1} f(s) d s,
$$

where $\Gamma(\cdot)$ is the Euler gamma function.
Definition 13 (see [31]). The Riemann-Liouville fractional derivative of order $\alpha>0, n-1<\alpha<n, n \in \mathbb{N}$ is defined as

$$
D_{0^{+}}^{\alpha} f(t)=\frac{1}{\Gamma(n-\alpha)}\left(\frac{d}{d t}\right)^{n} \int_{0}^{t}(t-s)^{n-\alpha-1} f(s) d s,
$$

where the function $f(t)$ has absolutely continuous derivatives up to order $(n-1)$.

Lemma 14 (see [31]). The equality $D_{0^{+}}^{\gamma} I_{0^{+}}^{\gamma} f(t)=f(t), \gamma>0$ holds for $f \in L^{1}(0,1)$.

Lemma 15 (see [31]). Let $\alpha>0$ and $u \in C(0,1) \cap L^{1}(0,1)$. Then, the differential equation

$$
D_{0^{+}}^{\alpha} u(t)=0
$$

has a unique solution $u(t)=c_{1} t^{\alpha-1}+c_{2} t^{\alpha-2}+\cdots+c_{n} t^{\alpha-n}, c_{i} \in \mathbb{R}$, $i=1, \ldots, n$, where $n-1<\alpha<n$.

Lemma 16 (see [31]). Let $\alpha>0$. Then, the following equality holds for $u \in L^{1}(0,1), D_{0^{+}}^{\alpha} u \in L^{1}(0,1)$ :

$$
I_{0^{+}}^{\alpha} D_{0^{+}}^{\alpha} u(t)=u(t)+c_{1} t^{\alpha-1}+c_{2} t^{\alpha-2}+\cdots+c_{n} t^{\alpha-n},
$$

$c_{i} \in \mathbb{R}, i=1, \ldots, n$, where $n-1<\alpha \leq n$.

By $A C^{1}([0,+\infty), \mathbb{R})$ we denote the space of continuous real-valued functions whose first derivative exists and it is absolutely continuous on $[0,+\infty)$. In this paper, we will use the following space $E$ to the study (1) which is denoted by

$$
\begin{gathered}
E=\left\{u \in A C^{1}([0,+\infty), \mathbb{R}): \sup _{0 \leq t<+\infty} \frac{|u(t)|}{1+t^{\alpha-1}},\right. \\
\left.\sup _{0 \leq t<+\infty} \frac{\left|u^{\prime}(t)\right|}{1+t^{\alpha-1}}<+\infty\right\} .
\end{gathered}
$$

From [32], we know that $E$ is a Banach space equipped with the norm

$$
\|u\|=\max \left\{\sup _{0 \leq t<+\infty} \frac{|u(t)|}{1+t^{\alpha-1}}, \sup _{0 \leq t<+\infty} \frac{\left|u^{\prime}(t)\right|}{1+t^{\alpha-1}}\right\} .
$$

In what follows, $I=[0,+\infty), \alpha \in(2,3)$, and $\Delta=$ $\sum_{i=1}^{m-2} \beta_{i} \xi_{i}^{\alpha-1}$. Next, we need the following technical result proved in [1].

Lemma 17 (see $[1])$. For any $h \in L^{1}([0,+\infty), \mathbb{R})$, the problem

$$
\begin{gathered}
D_{0^{+}}^{\alpha} u(t)+h(t)=0, \quad 0<t<\infty, 2<\alpha<3, \\
u(0)=u^{\prime}(0)=0, \quad D^{\alpha-1} u(+\infty)=\sum_{i=1}^{m-2} \beta_{i} u\left(\xi_{i}\right),
\end{gathered}
$$

has a unique solution $u(t)$ that

$$
u(t)=\int_{0}^{+\infty} G(t, s) h(s) d s,
$$


where

$$
\begin{gathered}
G(t, s)=G_{1}(t, s)+G_{2}(t, s), \\
G_{1}(t, s)=\frac{1}{\Gamma(\alpha)} \begin{cases}t^{\alpha-1}-(t-s)^{\alpha-1}, & 0 \leq s \leq t<+\infty \\
t^{\alpha-1}, & 0 \leq t \leq s<+\infty\end{cases} \\
G_{2}(t, s)=\frac{\sum_{i=1}^{m-2} \beta_{i} t^{\alpha-1}}{\Gamma(\alpha)-\Delta} G_{1}\left(\xi_{i}, s\right) .
\end{gathered}
$$

Note that $G(t, s)>0, \forall t, s \in[0,+\infty)$, (e.g., Lemma 3.2 in [1]) and from the definition of $G_{1}(t, s)$, we have the following (e.g., Remark 3.1 in [1]):

$$
\frac{G_{1}(t, s)}{1+t^{\alpha-1}} \leq \frac{1}{\Gamma(\alpha)}
$$

$$
\frac{G(t, s)}{1+t^{\alpha-1}} \leq L_{1} \quad \text { for }(t, s) \in[0,+\infty) \times[0,+\infty),
$$

where

$$
L_{1}=\frac{1}{\Gamma(\alpha)}\left(1+\frac{\sum_{i=1}^{m-2} \beta_{i} \xi_{m-2}^{\alpha-1}}{(\Gamma(\alpha)-\Delta)}\right) .
$$

Also, one can get

$$
\frac{\partial G(t, s) / \partial t}{1+t^{\alpha-1}} \leq L_{2} \quad \text { for }(t, s) \in[0,+\infty) \times[0,+\infty),
$$

where

$$
L_{2}=\frac{2(\alpha-1)}{\Gamma(\alpha)}\left(1+\frac{\sum_{i=1}^{m-2} \beta_{i} \xi_{m-2}^{\alpha-1}}{(\Gamma(\alpha)-\Delta)}\right) .
$$

Lemma 18. The function $G(t, s)$ defined by (16) satisfies

$$
\lim _{t \rightarrow+\infty} \frac{G(t, s)}{1+t^{\alpha-1}}=\frac{\sum_{i=1}^{m-2} \beta_{i} \xi_{i}^{\alpha-1}}{\Gamma(\alpha)(\Gamma(\alpha)-\Delta)} .
$$

By calculation, it is easy to prove that Lemma 18 holds. So, we omit its proof here.

\section{Main Results}

Now we are able to present the existence results for problem (1).

3.1. The Upper Semicontinuous Case. To obtain the complete continuity of existence solutions operator, the following lemma is still needed.

Lemma 19 (see [32]). Let $V=\{u \in E \mid\|u\|<l\}(l>$ $0), V_{1}=\left\{u(t) /\left(1+t^{\alpha-1}\right): u \in V\right\}$. If $V_{1}$ is equicontinuous on any compact interval of $[0,+\infty)$ and equiconvergent at infinity, then $V$ is relatively compact on $E$.

Definition 20. $V_{1}$ is called equiconvergent at infinity if and only if for all $\epsilon>0$, there exists $v(\epsilon)>0$ such that for all $u \in V_{1}, t_{1}, t_{2} \geq v$, it holds

$$
\left|\frac{u\left(t_{1}\right)}{1+t_{1}^{\alpha-1}}-\frac{u\left(t_{2}\right)}{1+t_{2}^{\alpha-1}}\right|<\epsilon .
$$

Theorem 21. The Carathéodory multivalued map $F: I \times$ $\mathbb{R} \times \mathbb{R} \rightarrow \mathscr{P}(\mathbb{R})$ has nonempty, compact, convex values and satisfies the following.

(H1) There exists a continuous nondecreasing function $\psi$ : $[0,+\infty) \rightarrow(0,+\infty)$ and $\varphi \in L^{1}\left([0,+\infty), \mathbb{R}^{+}\right)$ such that $\|F(t, x, y)\|_{\mathscr{P}}:=\sup \left\{\left|v(t) /\left(1+t^{\alpha-1}\right)\right|\right.$ : $v \in F(t, x, y)\} \leq \varphi(t) \psi(\|x\|)$, for a.e. $t \in$ $I$ and each $x, y \in \mathbb{R}$.

(H2) There exists a constant $M>0$ such that

$$
\frac{M}{\max \left\{L_{1}, L_{2}\right\} \psi(M) \int_{0}^{+\infty} \varphi(s) d s}>1 .
$$

Then, problem (1) has at least one solution.

Proof. Let $X=E$ and consider $M>0$ as in (25). It is obvious that the existence of solutions to problem (1) is reduced to the existence of the solutions of the integral inclusion

$$
u(t) \in \int_{0}^{+\infty} G(t, s) F\left(s, u(s), u^{\prime}(s)\right) d s, \quad t \in I
$$

where $G(t, s)$ is defined by (16) and (17). Consider the setvalued map, $T: E \rightarrow \mathscr{P}(X)$ is defined by

$$
T(u):=\left\{v \in X ; v(t)=\int_{0}^{+\infty} G(t, s) f(s) d s, f \in \overline{S_{F}(u)}\right\} .
$$

We show that $T$ satisfies the hypotheses of Theorem 2 .

Claim 1. We show that $T(u) \subset X$ is convex for any $u \in X$. If $v_{1}, v_{2} \in T(u)$, then, there exist $f_{1}, f_{2} \in S_{F}(u)$ such that for any $t \in I$ one has

$$
v_{i}(t)=\int_{0}^{+\infty} G(t, s) f_{i}(s) d s, \quad i=1,2 .
$$

Let $0 \leq \lambda \leq 1$. Then, for any $t \in I$, we have

$$
\begin{aligned}
\left(\lambda v_{1}\right. & \left.+(1-\lambda) v_{2}\right)(t) \\
& =\int_{0}^{+\infty} G(t, s)\left[\lambda f_{1}(s)+(1-\lambda) f_{2}(s)\right] d s .
\end{aligned}
$$

The values of $F$ are convex; thus, $S_{F}(u)$ is a convex set and hence $\lambda v_{1}+(1-\lambda) v_{2} \in T(u)$.

Claim 2. We show that $T$ is bounded on bounded sets of $X$. Let $B$ be any bounded subset of $X$. Then, there exists $m>0$ such that $\|u\| \leq m$ for all $u \in B$. If $v \in T(u)$, then there exists $f \in S_{F}(u)$ such that $v(t)=\int_{0}^{+\infty} G(t, s) f(s) d s$. One may write the following for any $t \in I$ :

$$
\begin{aligned}
\left|\frac{v(t)}{1+t^{\alpha-1}}\right| & \leq \int_{0}^{+\infty}\left|\frac{G(t, s)}{1+t^{\alpha-1}}\right||f(s)| d s \\
& \leq L_{1} \int_{0}^{+\infty} \varphi(s) \psi(\|u\|) d s \leq L_{1}|\varphi|_{1} \psi(m) .
\end{aligned}
$$


On the other hand,

$$
\begin{aligned}
\left|\frac{v^{\prime}(t)}{1+t^{\alpha-1}}\right| & \leq \int_{0}^{+\infty} \frac{\partial G(t, s) / \partial t}{1+t^{\alpha-1}} f(s) d s \\
& \leq L_{2} \int_{0}^{+\infty} \varphi(s) \psi(\|u\|) d s \\
& \leq L_{2}|\varphi|_{1} \psi(m),
\end{aligned}
$$

and therefore

$$
\begin{aligned}
\|v\| & =\max _{t \in I}\left\{\left|\frac{v(t)}{1+t^{\alpha-1}}\right|,\left|\frac{v^{\prime}(t)}{1+t^{\alpha-1}}\right|\right\} \\
& \leq \max \left\{L_{1}, L_{2}\right\}|\varphi|_{1} \psi(m),
\end{aligned}
$$

for all $v \in T(u)$; that is, $T(B)$ is bounded.

Claim 3. We show that $T$ maps bounded the sets into equicontinuous sets. Let $B$ be any bounded subset of $X$ as before and $v \in T(u)$ for some $u \in B$. Then, there exists $f \in S_{F}(u)$ such that $v(t)=\int_{0}^{+\infty} G(t, s) f(s) d s$. So, for any $T_{0} \in(0,+\infty)$ and $t_{1}, t_{2} \in\left[0, T_{0}\right]$, without loss of generality, we may assume that $t_{2}>t_{1}$ and one can get the following:

$$
\begin{aligned}
& \left|\frac{v\left(t_{1}\right)}{1+t_{1}^{\alpha-1}}-\frac{v\left(t_{2}\right)}{1+t_{2}^{\alpha-1}}\right| \\
& \leq \int_{0}^{+\infty}\left|\frac{G_{1}\left(t_{1}, s\right)}{1+t_{1}^{\alpha-1}}-\frac{G_{1}\left(t_{2}, s\right)}{1+t_{2}^{\alpha-1}}\right| f(s) d s \\
& \quad+\frac{\sum_{i=1}^{m-2} \beta_{i}}{\Gamma(\alpha)-\Delta}\left|\frac{t_{1}^{\alpha-1}}{1+t_{1}^{\alpha-1}}-\frac{t_{2}^{\alpha-1}}{1+t_{2}^{\alpha-1}}\right| \\
& \quad \times \int_{0}^{+\infty} G_{1}\left(\xi_{i}, s\right) f(s) d s \\
& \leq \int_{0}^{+\infty}\left|\frac{G_{1}\left(t_{1}, s\right)}{1+t_{1}^{\alpha-1}}-\frac{G_{1}\left(t_{2}, s\right)}{1+t_{1}^{\alpha-1}}\right| \varphi(s) \psi(\|u\|) d s \\
& \quad+\int_{0}^{+\infty}\left|\frac{G_{1}\left(t_{2}, s\right)}{1+t_{1}^{\alpha-1}}-\frac{G_{1}\left(t_{2}, s\right)}{1+t_{2}^{\alpha-1}}\right| \varphi(s) \psi(\|u\|) d s \\
& \quad+\frac{\sum_{i=1}^{m-2} \beta_{i} \xi_{m-2}^{\alpha-1}}{\Gamma(\Gamma(\alpha)-\Delta}\left|\frac{t_{1}^{\alpha-1}}{1+t_{1}^{\alpha-1}}-\frac{t_{2}^{\alpha-1}}{1+t_{2}^{\alpha-1}}\right| \\
& \quad \times \int_{0}^{+\infty} \varphi(s) \psi(\|u\|) d s .
\end{aligned}
$$

On the other hand, we get

$$
\begin{aligned}
& \int_{0}^{+\infty}\left|\frac{G_{1}\left(t_{1}, s\right)}{1+t_{1}^{\alpha-1}}-\frac{G_{1}\left(t_{2}, s\right)}{1+t_{1}^{\alpha-1}}\right| \varphi(s) \psi(\|u\|) d s \\
& \leq\left(\int_{0}^{t_{1}}+\int_{t_{1}}^{t_{2}}+\int_{t_{2}}^{+\infty}\right)\left|\frac{G_{1}\left(t_{1}, s\right)}{1+t_{1}^{\alpha-1}}-\frac{G_{1}\left(t_{2}, s\right)}{1+t_{1}^{\alpha-1}}\right| \\
& \quad \times \varphi(s) \psi(\|u\|) d s \\
& \leq \psi(m) \int_{0}^{t_{1}} \frac{\left(t_{2}^{\alpha-1}-t_{1}^{\alpha-1}\right)+\left(\left(t_{2}-s\right)^{\alpha-1}-\left(t_{1}-s\right)^{\alpha-1}\right)}{1+t_{1}^{\alpha-1}} \\
& \times \varphi(s) d s
\end{aligned}
$$

$$
\begin{aligned}
& +\psi(m) \int_{t_{1}}^{t_{2}} \frac{\left(t_{2}^{\alpha-1}-t_{1}^{\alpha-1}\right)+\left(t_{2}-s\right)^{\alpha-1}}{1+t_{1}^{\alpha-1}} \varphi(s) d s \\
& +\psi(m) \int_{t_{2}}^{+\infty} \frac{\left(t_{2}^{\alpha-1}-t_{1}^{\alpha-1}\right)}{1+t_{1}^{\alpha-1}} \varphi(s) d s
\end{aligned}
$$$$
\longrightarrow 0 \text { uniformly as } t_{1} \longrightarrow t_{2} \text {. }
$$

Similar to (34), we have

$$
\begin{array}{r}
\int_{0}^{+\infty}\left|\frac{G_{1}\left(t_{2}, s\right)}{1+t_{1}^{\alpha-1}}-\frac{G_{1}\left(t_{2}, s\right)}{1+t_{2}^{\alpha-1}}\right| \varphi(s) \psi(\|u\|) d s \longrightarrow 0 \\
\text { uniformly as } t_{1} \longrightarrow t_{2} .
\end{array}
$$

From (34) and (35), we have

$$
\left|\frac{v\left(t_{1}\right)}{1+t_{1}^{\alpha-1}}-\frac{v\left(t_{2}\right)}{1+t_{2}^{\alpha-1}}\right| \longrightarrow 0 \quad \text { uniformly as } t_{1} \longrightarrow t_{2} .
$$

Similar to (36), one can get

$$
\left|\frac{v^{\prime}\left(t_{1}\right)}{1+t_{1}^{\alpha-1}}-\frac{v^{\prime}\left(t_{2}\right)}{1+t_{2}^{\alpha-1}}\right| \longrightarrow 0 \quad \text { uniformly as } t_{1} \longrightarrow t_{2} .
$$

Therefore, $T(B)$ is an equicontinuous set in $X$.

Claim 4. We show that $T$ is equiconvergent at $\infty$. Let $v \in T(u)$ for some $u \in B$. Then, there exists $f \in S_{F}(u)$ such that $v(t)=$ $\int_{0}^{+\infty} G(t, s) f(s) d s$. So, we have the following:

$$
\begin{aligned}
\int_{0}^{+\infty} f(s) d s & \leq \psi(m) \int_{0}^{+\infty} \varphi(s) d s<+\infty \\
\lim _{t \rightarrow+\infty}\left|\frac{v(t)}{1+t^{\alpha-1}}\right|= & \lim _{t \rightarrow+\infty} \frac{1}{1+t^{\alpha-1}} \int_{0}^{+\infty} G(t, s) f(s) d s \\
= & \frac{\sum_{i=1}^{m-2} \beta_{i} \xi_{i}^{\alpha-1}}{\Gamma(\alpha)(\Gamma(\alpha)-\Delta)} \int_{0}^{+\infty} f(s) d s \\
& -\frac{\sum_{i=1}^{m-2} \beta_{i}}{\Gamma(\alpha)(\Gamma(\alpha)-\Delta)} \\
& \times \int_{0}^{\xi_{i}}\left(\xi_{i}-s\right)^{\alpha-1} f(s) d s<\infty,
\end{aligned}
$$


and, similarly, one has

$$
\lim _{t \rightarrow+\infty}\left|\frac{v^{\prime}(t)}{1+t^{\alpha-1}}\right|<\infty .
$$

Therefore, $T(B)$ is equiconvergent at infinity.

Therefore, with Lemma 11, Lemma 19 and Claims 2-4, we conclude that $T$ is completely continuous.

Claim 5. $T$ is upper semicontinuous. To this end, it is sufficient to show that $T$ has a closed graph. Let $v_{n} \in T\left(u_{n}\right)$ such that $v_{n} \rightarrow v$ and $u_{n} \rightarrow u$, as $n \rightarrow+\infty$. Then, there exists $m>0$ such that $\left\|u_{n}\right\| \leq m$. We will prove that $v \in T(u)$ means that there exists $f_{n} \in S_{F}\left(u_{n}\right)$ such that, for a.e. $t \in I$, we have $v_{n}(t)=\int_{0}^{+\infty} G(t, s) f_{n}(s) d s$. Then, we need to show that $v \in$ $T(u)$.

Condition (H1) implies that $f_{n}(t) \in \varphi(t) \psi(m) B_{1}(0)$. Then, $\left\{f_{n}\right\}_{n \in \mathbb{N}}$ is integrably bounded in $L^{1}(I, \mathbb{R})$. Since $F$ has compact values, we deduce that $\left\{f_{n}\right\}_{n}$ is semicompact. By Lemma 9, there exists a subsequence, still denoted as $\left\{f_{n}\right\}_{n \in \mathbb{N}}$, which converges weakly to some limit $f \in L^{1}(I, \mathbb{R})$. Moreover, the mapping $\Gamma: L^{1}(I, \mathbb{R}) \rightarrow X=E$ defined by

$$
\Gamma(g)(t)=\int_{0}^{+\infty} G(t, s) g(s) d s
$$

is a continuous linear operator. Then, it remains continuous if these spaces are endowed with their weak topologies $[29,33]$. Moreover, for a.e. $t \in I, u_{n}(t)$ converges to $u(t)$. Then, we have

$$
v(t)=\int_{0}^{+\infty} G(t, s) f(s) d s .
$$

It remains to prove that $f \in F\left(t, u(t), u^{\prime}(t)\right)$, a.e. $t \in I$. Mazur's Lemma (see Lemma 10) yields the existence of $\alpha_{i}^{n} \geq 0, i=$ $n, \ldots, k(n)$ such that $\sum_{i=1}^{k(n)} \alpha_{i}^{n}=1$ and the sequence of convex combinations $g_{n}(\cdot)=\sum_{i=1}^{k(n)} \alpha_{i}^{n} f_{i}(\cdot)$ converges strongly to $f$ in $L^{1}$. Using Lemma 6 , we obtain that

$$
\begin{aligned}
v(t) & \in \bigcap_{n \geq 1} \overline{\left\{g_{n}(t)\right\}}, \quad \text { a.e. } t \in I \\
& \subset \bigcap_{n \geq 1} \overline{\operatorname{co}}\left\{f_{k}(t), k \geq n\right\} \\
& \subset \bigcap_{n \geq 1} \overline{\operatorname{co}}\left\{\bigcup_{n \geq 1} F\left(t, u_{k}(t), u_{k}^{\prime}(t)\right)\right\} \\
& =\overline{c o}\left(\limsup _{k \rightarrow+\infty} F\left(t, u_{k}(t), u_{k}^{\prime}(t)\right)\right) .
\end{aligned}
$$

However, the fact that the multivalued $x \rightarrow F\left(\cdot, x, x^{\prime}\right)$ is upper semicontinuous and has compact values, together with Lemma 7, implies that

$$
\limsup _{n \rightarrow+\infty} F\left(t, u_{n}(t), u_{n}^{\prime}(t)\right)=F\left(t, u(t), u^{\prime}(t)\right), \quad \text { a.e. } t \in I .
$$

This along with (42) yields that $f(t) \in \overline{\mathrm{co}} F\left(t, u(t), u^{\prime}(t)\right)$. Finally, $F(\cdot, \cdot, \cdot)$ has closed, convex values; hence, $f(t) \in$
$F\left(t, u(t), u^{\prime}(t)\right)$, a.e. $t \in I$. Thus, $v \in T(u)$, proving that $T$ has a closed graph. Finally, with Lemma 1 and the compactness of $T$, we conclude that $T$ is upper semicontinuous.

Claim 6. A priori bounds on solutions. Let $u$ be a solution of (1). Then, there exists $f \in L^{1}([0,+\infty), \mathbb{R})$ with $f \in S_{F}(u)$ such that $u(t)=\int_{0}^{+\infty} G(t, s) f(s) d s$. In view of $(\mathrm{H} 1)$, and using the computations in Claim 2 above, for each $t \in[0,+\infty)$, we obtain

$$
\begin{aligned}
\left\{\left|\frac{u(t)}{1+t^{\alpha-1}}\right|,\left|\frac{u^{\prime}(t)}{1+t^{\alpha-1}}\right|\right\} \leq & \max \left\{L_{1}, L_{2}\right\} \int_{0}^{+\infty} f(s) d s \\
\leq & \max \left\{L_{1}, L_{2}\right\} \psi(\|u\|) \\
& \times \int_{0}^{+\infty} \varphi(s) d s .
\end{aligned}
$$

Consequently,

$$
\frac{\|u\|}{\max \left\{L_{1}, L_{2}\right\} \psi(\|u\|) \int_{0}^{+\infty} \varphi(s) d s} \leq 1 .
$$

In view of $(\mathrm{H} 2)$, there exists $M$ such that $\|u\| \neq M$. Let us consider the following:

$$
U:=\left\{u \in A C^{1}([0,+\infty), \mathbb{R}):\|u\|<M\right\} .
$$

Note that the operator $T: \bar{U} \rightarrow \mathscr{P}\left(A C^{1}([0,+\infty))\right.$ is upper semicontinuous and completely continuous. From the choice of $U$, there is no $u \in \partial U$ such that $u=\lambda T(u)$ for some $\lambda \in(0,1)$. Consequently, by the nonlinear alternative of the Leray-Schauder type (Theorem 2), we deduce that $T$ has a fixed point $u \in U$ which is a solution of the problem (1). This completes the proof.

3.2. The Lipschitz Case. Now we prove the existence of solutions for the problem (1) with a nonconvex-valued right hand side by applying a fixed point theorem for multivalued maps according to Covitz and Nadler [34].

Definition 22. A multivalued operator $N: X \rightarrow \mathscr{P}_{c l}(X)$ is called the following:

(a) $\gamma$-Lipschitz if and only if there exists $\gamma>0$ such that $d_{H}(N(x), N(y)) \leq \gamma d(x, y)$ for each $x, y \in X$;

(b) a contraction if and only if it is $\gamma$-Lipschitz with $\gamma<1$.

Lemma 23 (Covitz-Nadler, [34]). Let $(X, d)$ be a complete metric space. If $N: X \rightarrow \mathscr{P}_{c l}(X)$ is a contraction, then Fix $N \neq \emptyset$.

Definition 24. A measurable multivalued function $F$ : $[0,+\infty) \rightarrow \mathscr{P}(X)$ is said to be integrably bounded if there exists a function $f \in L^{1}([0,+\infty), X)$ such that for all $v \in F(t)$, $\|v\| \leq f(t)$ for a.e. $t \in[0,+\infty)$.

Theorem 25. Assume that the following condition holds:

(H4) $F: I \times \mathbb{R} \times \mathbb{R} \rightarrow \mathscr{P}_{c p}(\mathbb{R})$ such that $F(\cdot, x, y):$ $[0,+\infty) \rightarrow \mathscr{P}_{c p}(\mathbb{R})$ is measurable for each $x, y \in \mathbb{R} ;$ 
(H5) There exist $l_{1}, l_{2}:[0,+\infty) \rightarrow[0,+\infty)$ which are not identical zero on any closed subinterval of $[0,+\infty)$, and

$$
\int_{0}^{+\infty}\left(1+s^{\alpha-1}\right) l_{i}(s) d s<+\infty, \quad i=1,2,
$$

such that for almost all $t \in[0,+\infty)$,

$$
\begin{aligned}
d_{H}\left(F\left(t, x_{1}, y_{1}\right), F\left(t, x_{2}, y_{2}\right)\right) \leq & l_{1}(t)\left|x_{1}-x_{2}\right| \\
& +l_{2}(t)\left|y_{1}-y_{2}\right|
\end{aligned}
$$

for all $x_{1}, x_{2}, y_{1}$, and $y_{2} \in \mathbb{R}$ with $d(0, F(t, 0,0)) \leq l_{1}(t)+l_{2}(t)$ for almost all $t \in[0,+\infty)$.

Then, the boundary value problem (1) has at least one solution on $I=[0,+\infty)$ if

$$
\max \left\{L_{1}, L_{2}\right\}\left(\int_{0}^{+\infty}\left(1+s^{\alpha-1}\right)\left(l_{1}(s)+l_{2}(s)\right) d s\right)<1 .
$$

Proof. We transform problem (1) into a fixed point problem. Consider the set-valued map $T: A C^{1}[0,+\infty) \rightarrow$ $\mathscr{P}\left(A C^{1}[0,+\infty)\right)$ defined at the beginning of the proof of Theorem 21. It is clear that the fixed point of $T$ are solutions of the problem (1).

Note that since the set-valued map $F(\cdot, u(\cdot))$ is measurable with the measurable selection theorem (e.g., Theorem III. 6 in [35]) it admits a measurable selection $f: I \rightarrow \mathbb{R}$. Moreover, since $F$ is integrably bounded, $f \in L^{1}([0,+\infty), \mathbb{R})$. Therefore, $S_{F}(u) \neq \emptyset$.

We will prove that $T$ fulfills the assumptions of CovitzNadler contraction principle (Lemma 23).

First, we note that since $S_{F}(u) \neq \emptyset, T(u) \neq \emptyset$ for any $u \in$ $A C^{1}([0,+\infty), \mathbb{R})$.

Second, we prove that $T(u)$ is closed for any $u \in$ $A C^{1}([0,+\infty), \mathbb{R})$. Let $\left\{u_{n}\right\}_{n \geq 0} \in T(u)$ such that $u_{n} \rightarrow u_{0}$ in $A C^{1}([0,+\infty), \mathbb{R})$. Then $u_{0} \in A C^{1}([0,+\infty), \mathbb{R})$ and there exists $f_{n} \in S_{F}(u)$ such that

$$
u_{n}(t)=\int_{0}^{+\infty} G(t, s) f_{n}(s) d s .
$$

Since $F$ has compact values, we may pass onto a subsequence (if necessary) to obtain that $f_{n}$ converges to $f \in$ $L^{1}(([0,+\infty), \mathbb{R}))$ in $L^{1}(([0,+\infty), \mathbb{R}))$. In particular, $f \in$ $S_{F}(u)$, and for any $t \in[0,+\infty)$ we have

$$
u_{n}(t) \longrightarrow u_{0}(t)=\int_{0}^{+\infty} G(t, s) f(s) d s,
$$

that is, $u_{0} \in T(u)$ and $T(u)$ is closed.

Next we show that $T$ is a contraction on $A C^{1}([0,+\infty), \mathbb{R})$. Let $u_{1}, u_{2} \in A C^{1}([0,+\infty), \mathbb{R})$ and $v_{1} \in T\left(u_{1}\right)$. Then there exist $f_{1} \in S_{F}\left(u_{1}\right)$ such that

$$
v_{1}(t)=\int_{0}^{+\infty} G(t, s) f_{1}(s) d s, \quad t \in[0,+\infty) .
$$

Consider the set-valued map

$$
\begin{aligned}
H(t):= & F\left(t, u_{2}(t), u_{2}^{\prime}(t)\right) \\
\cap\{u \in \mathbb{R} ; & \left|f_{1}(t)-u\right| \leq l_{1}(t)\left|x_{1}-x_{2}\right| \\
& \left.\quad+l_{2}(t)\left|x_{1}^{\prime}-x_{2}^{\prime}\right|\right\}, \quad t \in[0,+\infty) .
\end{aligned}
$$

By (H5), we have

$$
\begin{aligned}
d_{H}\left(F\left(t, x_{1}, x_{1}^{\prime}\right), F\left(t, x_{2}, x_{2}^{\prime}\right)\right) \leq & l_{1}(t)\left|x_{1}-x_{2}\right| \\
& +l_{2}(t)\left|x_{1}^{\prime}-x_{2}^{\prime}\right|,
\end{aligned}
$$

hence $H$ has nonempty closed values. Moreover, since $H$ is measurable (e.g., Proposition III. 4 in [35]), there exists $f_{2}$ which is a measurable selection of $H$. It follows that $f_{2} \in$ $S_{F}\left(u_{2}\right)$ and for any $t \in[0,+\infty)$,

$$
\left|f_{1}(t)-f_{2}(t)\right| \leq l_{1}(t)\left|x_{1}-x_{2}\right|+l_{2}(t)\left|x_{1}^{\prime}-x_{2}^{\prime}\right| .
$$

Define

$$
v_{2}(t)=\int_{0}^{+\infty} G(t, s) f_{2}(s) d s, \quad t \in[0,+\infty),
$$

and one can get

$$
\begin{aligned}
& \left|\frac{v_{1}(t)}{1+t^{\alpha-1}}-\frac{v_{2}(t)}{1+t^{\alpha-1}}\right| \\
& \leq \int_{0}^{+\infty} \frac{G(t, s)}{1+t^{\alpha-1}}\left|f_{1}(s)-f_{1}(s)\right| d s \\
& \leq L_{1} \int_{0}^{+\infty}\left[l_{1}(s)\left|x_{1}(s)-x_{2}(s)\right|+l_{2}(s)\left|x_{1}^{\prime}(s)-x_{2}^{\prime}(s)\right|\right] d s \\
& \leq L_{1} \int_{0}^{+\infty}\left(1+s^{\alpha-1}\right)\left[l_{1}(s)\left|\frac{x_{1}(s)-x_{2}(s)}{1+s^{\alpha-1}}\right|\right. \\
& \left.+l_{2}(s)\left|\frac{x_{1}^{\prime}(s)-x_{2}^{\prime}(s)}{1+s^{\alpha-1}}\right|\right] d s \\
& \leq \max \left\{L_{1}, L_{2}\right\}\left\|x_{1}-x_{2}\right\| \int_{0}^{+\infty}\left(1+s^{\alpha-1}\right)\left(l_{1}(s)+l_{2}(s)\right) d s .
\end{aligned}
$$

Similarly, we have

$$
\begin{aligned}
\left|\frac{v_{1}^{\prime}(t)}{1+t^{\alpha-1}}-\frac{v_{2}^{\prime}(t)}{1+t^{\alpha-1}}\right| \leq & \max \left\{L_{1}, L_{2}\right\}\left\|x_{1}-x_{2}\right\| \\
& \times \int_{0}^{+\infty}\left(1+s^{\alpha-1}\right)\left(l_{1}(s)+l_{2}(s)\right) d s .
\end{aligned}
$$

Therefore,

$$
\begin{aligned}
\left\|v_{1}-v_{2}\right\| \leq & \max \left\{L_{1}, L_{2}\right\}\left\|x_{1}-x_{2}\right\| \\
& \times \int_{0}^{+\infty}\left(1+s^{\alpha-1}\right)\left(l_{1}(s)+l_{2}(s)\right) d s .
\end{aligned}
$$


From an analogous reasoning by interchanging the roles of $u_{1}$ and $u_{2}$, it follows that

$$
\begin{aligned}
d_{H}\left(T\left(u_{1}\right), T\left(u_{2}\right)\right) \leq & \max \left\{L_{1}, L_{2}\right\}\left\|x_{1}-x_{2}\right\| \\
& \times \int_{0}^{+\infty}\left(1+s^{\alpha-1}\right)\left(l_{1}(s)+l_{2}(s)\right) d s
\end{aligned}
$$

Since $T$ is a contraction, it follows by the Lemma 23 that $T$ admits a fixed point which is a solution to problem (1).

\section{Application}

Consider the fractional boundary value problem,

$$
\begin{gathered}
D_{0^{+}}^{5 / 2} u(t)+F\left(t, u(t), u^{\prime}(t)\right) \ni 0, \quad 0<t<+\infty \\
u(0)=u^{\prime}(0)=0, \quad D^{3 / 2} u(+\infty)-\frac{1}{8} u\left(\frac{1}{8}\right)-\frac{1}{4} u(1)=0 .
\end{gathered}
$$

Here $m=4, \alpha=5 / 2, \beta_{1}=1 / 8, \beta_{2}=1 / 4, \xi_{1}=1 / 8$, and $\xi_{2}=1$, and $F: I \times \mathbb{R} \times \mathbb{R} \rightarrow \mathscr{P}(\mathbb{R})$ is a multivalued map given by

$$
\begin{aligned}
F(t, x, y)= & {\left[e^{-t}\left(1+t^{3 / 2}\right)\left(\frac{|x+y|^{5}}{|x+y|^{5}+5}+9\right)\right.} \\
& \left.2 e^{-t}\left(1+t^{3 / 2}\right)\left(\frac{|x+y|^{3}}{|x+y|^{3}+3}+1\right)\right] .
\end{aligned}
$$

For $v \in F$, we have

$$
\begin{aligned}
\left|\frac{v(t)}{1+t^{3 / 2}}\right| \leq \max \left(e^{-t}\left(\frac{|x+y|^{5}}{|x+y|^{5}+5}+9\right)\right. \\
\left.\quad 2 e^{-t}\left(\frac{|x+y|^{3}}{|x+y|^{3}+3}+1\right)\right) \\
\leq 10 e^{-t}, \quad x, y \in \mathbb{R} .
\end{aligned}
$$

Thus,

$$
\begin{aligned}
\|F(t, x, y)\|_{\mathscr{P}} & :=\sup \left\{\left|\frac{v(t)}{1+t^{3 / 2}}\right|: v \in F(t, x, y)\right\} \\
& \leq 10 e^{-t}=\varphi(t) \psi(\|x\|), \quad x, y \in \mathbb{R},
\end{aligned}
$$

with $\varphi(t)=e^{-t}, \psi(\|x\|)=10$.

Also, by direct calculation, we can obtain that $L_{1}=$ 1.01529 and $L_{2}=3.045869$. Further, by using the following condition:

$$
\frac{M}{\max \left\{L_{1}, L_{2}\right\} \psi(M) \int_{0}^{+\infty} \varphi(s) d s}>1,
$$

we find that $M>30.45869$. Clearly, all the conditions of Theorem 21 are satisfied. So, there exists at least one solution of problem (1) on $I$.

\section{Acknowledgments}

The authors thank the referees for their careful reading of this paper and useful suggestions. This paper was funded by King Abdulaziz University, under Grant no. (130-1-1433/HiCi). The authors, therefore, acknowledge technical and financial support of KAU.

\section{References}

[1] S. Liang and J. Zhang, "Existence of multiple positive solutions for $m$-point fractional boundary value problems on an infinite interval," Mathematical and Computer Modelling, vol. 54, no. 56, pp. 1334-1346, 2011.

[2] A. M. A. El-Sayed, "Nonlinear functional-differential equations of arbitrary orders," Nonlinear Analysis. Theory, Methods \& Applications, vol. 33, no. 2, pp. 181-186, 1998.

[3] A. A. Kilbas and J. J. Trujillo, "Differential equations of fractional order: methods, results and problems. I," Applicable Analysis, vol. 78, no. 1-2, pp. 153-192, 2001.

[4] A. A. Kilbas and J. J. Trujillo, "Differential equations of fractional order: methods, results and problems. II," Applicable Analysis, vol. 81, no. 2, pp. 435-493, 2002.

[5] D. Baleanu, K. Diethelm, E. Scalas, and J. J. Trujillo, Fractional Calculus Models and Numerical Methods, vol. 3 of Series on Complexity, Nonlinearity and Chaos, World Scientific, Singapore, 2012.

[6] I. Podlubny, Fractional Differential Equations, vol. 198 of Mathematics in Science and Engineering, Academic Press, San Diego, Calif, USA, 1999.

[7] I. Podlubny, The Laplace Transform Method for Linear Differential Equations of Fractional Order, Slovac Academy of Science, Slovak Republic, 1994.

[8] S. G. Samko, A. A. Kilbas, and O. I. Marichev, Fractional Integrals and Derivatives: Theory and Applications, Gordon and Breach, Amsterdam, The Netherlands, 1993.

[9] R. P. Agarwal, M. Benchohra, and S. Hamani, "A survey on existence results for boundary value problems of nonlinear fractional differential equations and inclusions," Acta Applicandae Mathematicae, vol. 109, no. 3, pp. 973-1033, 2010.

[10] V. Lakshmikantham and A. S. Vatsala, "Basic theory of fractional differential equations," Nonlinear Analysis. Theory, Methods \& Applications, vol. 69, no. 8, pp. 2677-2682, 2008.

[11] N. Nyamoradi, "Existence of solutions for multi point boundary value problems for fractional differential equations," Arab Journal of Mathematical Sciences, vol. 18, no. 2, pp. 165-175, 2012.

[12] N. Nyamoradi, "Positive solutions for multi-point boundary value problem for nonlinear fractional differential equations," Journal of Contemporary Mathematical Analysis. Accepted for publishing.

[13] N. Nyamoradi, "A Six-point nonlocal integral boundary value problem for fractional differential equations," Indian Journal of Pure and Applied Mathematics, vol. 43, no. 5, pp. 429-454, 2012.

[14] N. Nyamoradi and T. Bashiri, "Multiple positive solutions for nonlinear fractional differential systems," Fractional Differential Calculus, vol. 2, no. 2, pp. 119-128, 2012.

[15] N. Nyamoradi and T. Bashiri, "Existence of positive solutions for fractional differential systems with multi point boundary conditions," Annali Dell'Universita di Ferrara, 2012.

[16] R. P. Agarwal and B. Ahmad, "Existence theory for anti-periodic boundary value problems of fractional differential equations 
and inclusions," Computers \& Mathematics with Applications, vol. 62, no. 3, pp. 1200-1214, 2011.

[17] B. Ahmad and S. K. Ntouyas, "Existence results for nonlocal boundary value problems of fractional differential equations and inclusions with strip conditions," Boundary Value Problems, vol. 2012, article 55, 21 pages, 2012.

[18] A. M. A. El-Sayed and A.-G. Ibrahim, "Multivalued fractional differential equations," Applied Mathematics and Computation, vol. 68, no. 1, pp. 15-25, 1995.

[19] B. Ahmad and S. K. Ntouyas, "Existence of solutions for fractional differential inclusions with nonlocal strip conditions," Arab Journal of Mathematical Sciences, vol. 18, no. 2, pp. 121-134, 2012.

[20] M. Benchohra, J. Henderson, S. K. Ntouyas, and A. Ouahab, "Existence results for fractional functional differential inclusions with infinite delay and applications to control theory," Fractional Calculus \& Applied Analysis, vol. 11, no. 1, pp. 35-56, 2008.

[21] A. Cernea, "Continuous version of Filippov's theorem for fractional differential inclusions," Nonlinear Analysis. Theory, Methods \& Applications, vol. 72, no. 1, pp. 204-208, 2010.

[22] N. Nyamoradi and M. Javidi, "Exictence of multiple positive soulutions for fractional differential inclusion with m-point boundary conditions and two fractional orders," Electronic Journal of Differential Equations, vol. 2012, p. 126, 2012.

[23] A. Ouahab, "Some results for fractional boundary value problem of differential inclusions," Nonlinear Analysis. Theory, Methods \& Applications, vol. 69, no. 11, pp. 3877-3896, 2008.

[24] K. Deimling, Multivalued Differential Equations, vol. 1 of de Gruyter Series in Nonlinear Analysis and Applications, Walter de Gruyter, Berlin, Germany, 1992.

[25] A. Granas and J. Dugundji, Fixed Point Theory, Springer Monographs in Mathematics, Springer, New York, NY, USA, 2003.

[26] A. Bressan and G. Colombo, "Extensions and selections of maps with decomposable values," Studia Mathematica, vol. 90, no. 1, pp. 70-85, 1988.

[27] J.-P. Aubin and H. Frankowska, Set-Valued Analysis, vol. 2 of Systems \& Control: Foundations \& Applications, Birkhäuser, Boston, Mass, USA, 1990.

[28] M. Kamenskii, V. Obukhovskii, and P. Zecca, Condensing Multivalued Maps and Semilinear Differential Inclusions in Banach Spaces, vol. 7 of De Gruyter Series in Nonlinear Analysis and Applications, Walter de Gruyter \& Co., Berlin, Germany, 2001.

[29] J. Musielak, Introduction to Functional Analysis, PWN, Warsaw, Poland, 1976.

[30] R. P. Agarwal and D. O'Regan, Infinite Interval Problems for Differential, Difference and Integral Equations, Kluwer Academic Publishers, Dordrecht, The Netherlands, 2001.

[31] A. A. Kilbas, H. M. Srivastava, and J. J. Trujillo, Theory and Applications of Fractional Differential Equations, vol. 204 of North-Holland Mathematics Studies, Elsevier Science, Amsterdam, The Netherlands, 2006.

[32] Y. Liu, "Existence and unboundedness of positive solutions for singular boundary value problems on half-line," Applied Mathematics and Computation, vol. 144, no. 2-3, pp. 543-556, 2003.

[33] L. V. Kantorovich and G. P. Akilov, Functional Analysis in Normed Spaces, The Macmillan, New York, NY, USA, 1964.

[34] H. Covitz and S. B. Nadler, Jr., "Multi-valued contraction mappings in generalized metric spaces," Israel Journal of Mathematics, vol. 8, pp. 5-11, 1970.
[35] C. Castaing and M. Valadier, Convex Analysis and Measurable Multifunctions, vol. 580 of Lecture Notes in Mathematics, Springer, Berlin, Germany, 1977. 


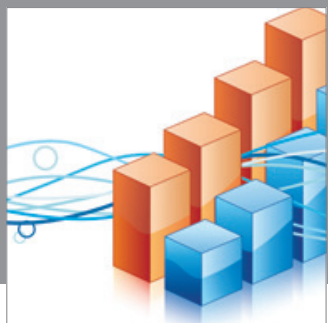

Advances in

Operations Research

mansans

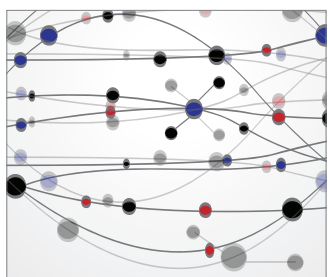

The Scientific World Journal
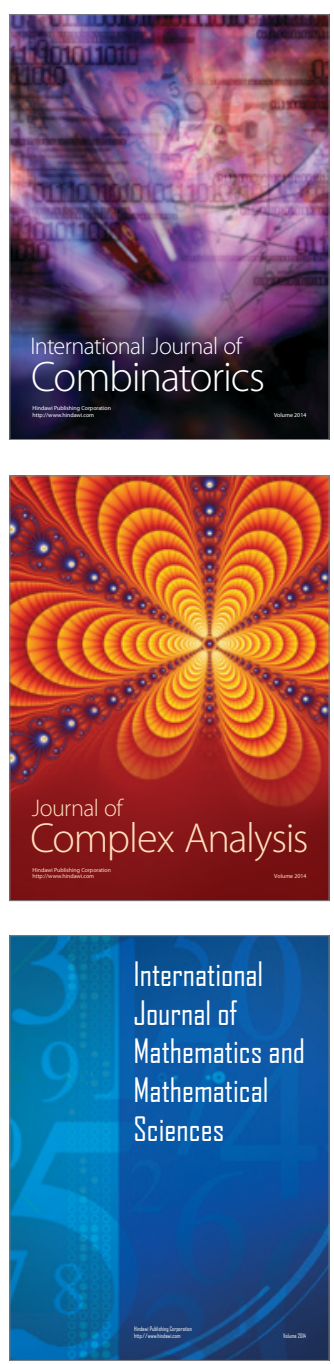
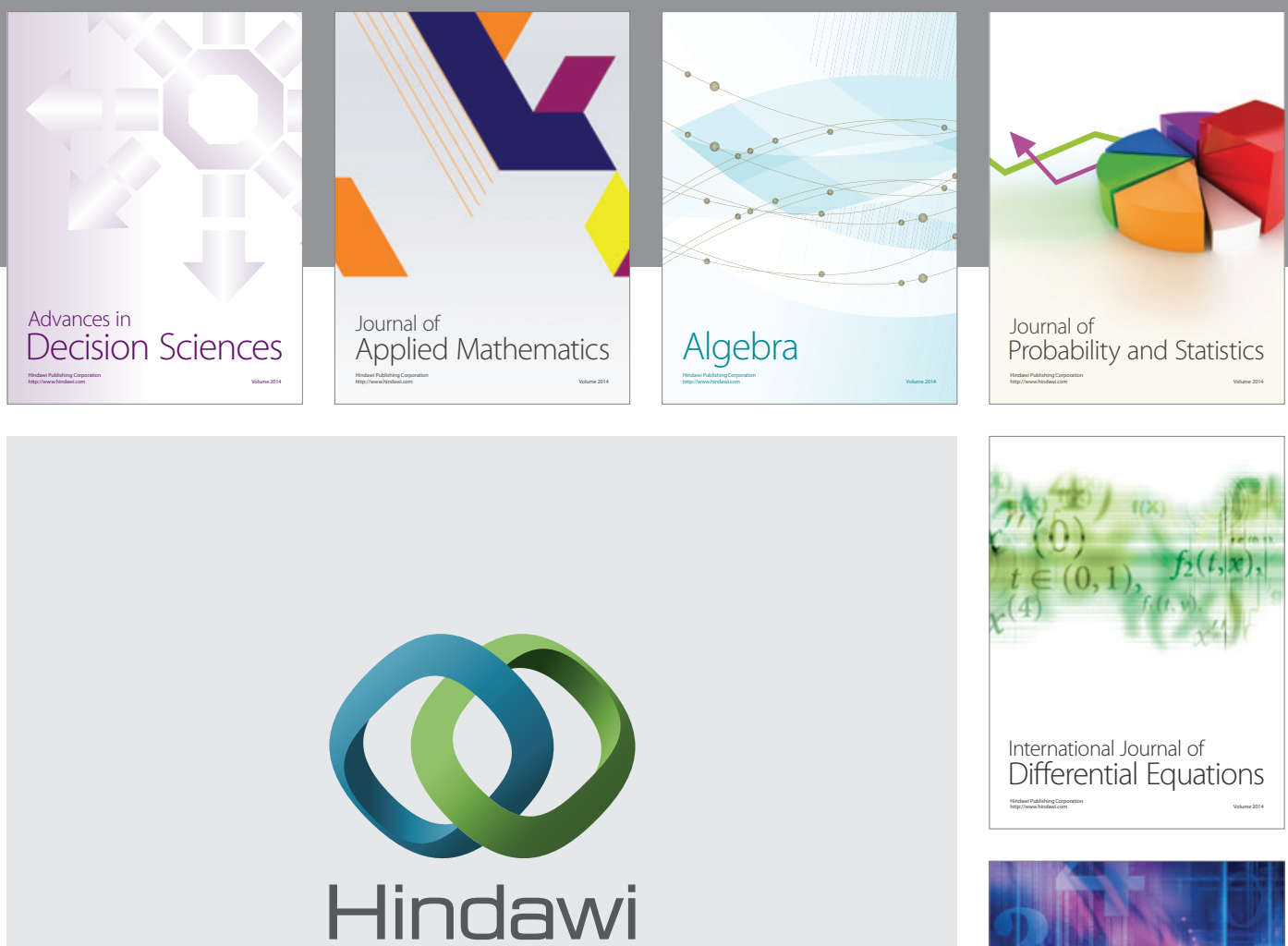

Submit your manuscripts at http://www.hindawi.com
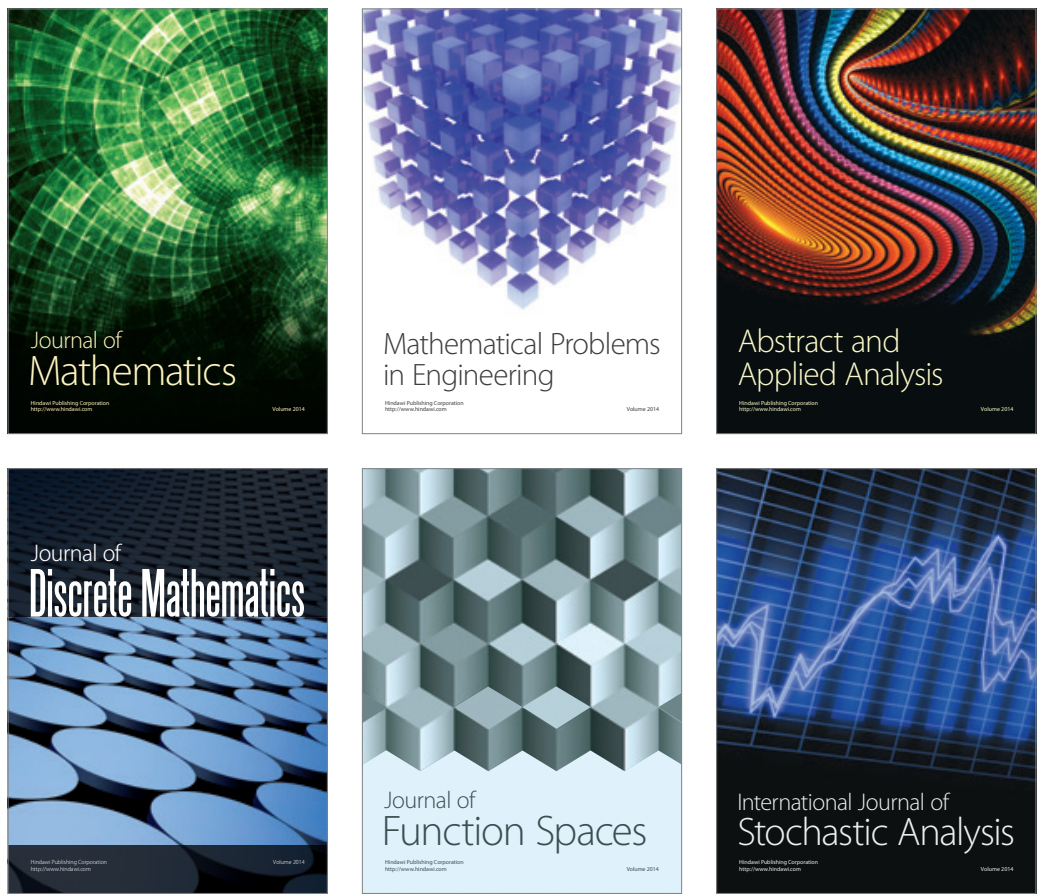

Journal of

Function Spaces

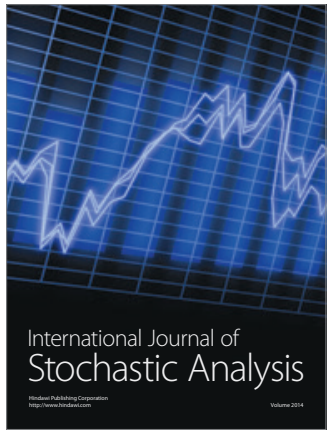

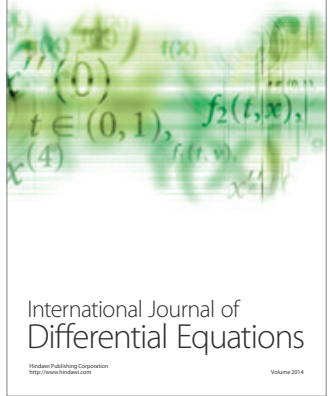
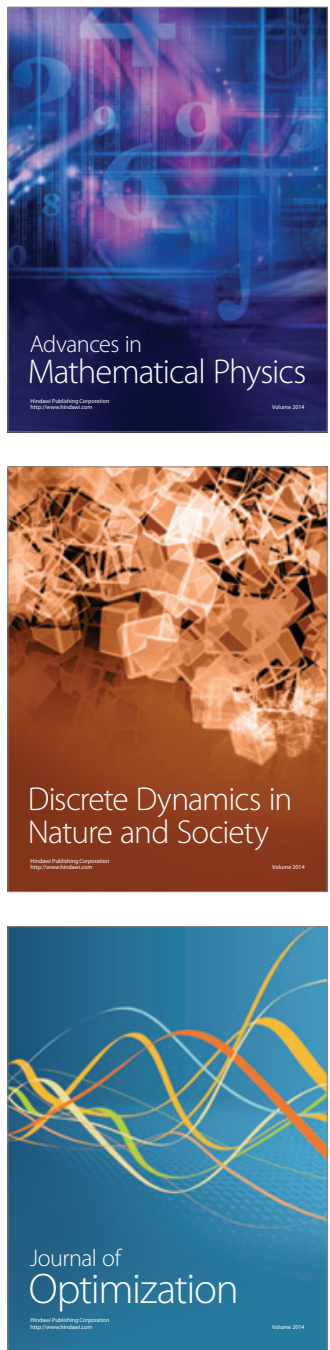